\title{
Regularity, positivity and asymptotic vanishing of solutions of a $\phi$-Laplacian
}

\author{
Waldo Arriagada and Jorge Huentutripay
}

\begin{abstract}
In this note we prove that solutions of a $\phi$-Laplacian operator on the entire space $\mathbb{R}^{N}$ are locally regular (Hölder continuous), positive and vanish at infinity. Mild restrictions are imposed on the right-hand side of the equation. For example, we assume a Lieberman-like condition but the hypothesis of differentiability is dropped. This is in striking contrast with the classical case.
\end{abstract}

\section{Introduction}

G. Lieberman addressed in [20] the problem on the regularity of solutions of quasilinear elliptic equations in the generic divergence form

$$
-\operatorname{div} A(x, u, D u)=B(x, u, D u) .
$$

The functionals $A$ and $B$ are governed by structural estimates which somewhat generalize the natural conditions introduced earlier on by Ladyzhenskaya and Ural'tseva in the 1960's [19, 18]. Lieberman introduces a generalized Harnack's inequality which is subsequently employed in the study of the behavior and regularity of the solutions of the differential equation.

The subject of this manuscript is motivated by the analysis of this kind of differential problems in the divergence form. The topic itself belongs to a vast program devoted to the study of the properties of the modified Laplace operator $\Delta_{\phi}$ (or $\phi$-Laplacian) defined by

$$
\Delta_{\phi} u=\operatorname{div}\left(\phi(|\nabla u|) \frac{\nabla u}{|\nabla u|}\right)
$$

Key Words: Orlicz-Sobolev space, $\phi$-Laplacian, regularity, asymptotic vanishing. 2010 Mathematics Subject Classification: Primary 35P30, 35J20; Secondary 46E30. 
where $\phi: \mathbb{R} \rightarrow \mathbb{R}$ is an odd, increasing and not-necessarily differentiable homeomorphism, see $[4,9,10]$. For example, in [3] we have treated the blow-up rates of large solutions of such $\phi$-Laplacians via a dynamical formulation. The latter provides a parallel analysis between the asymptotic properties of large solutions and the behavior of the orbits of an autonomous ordinary differential equation.

The existence of nontrivial solutions of equations in the divergence form is proved in [5]via a classical Lagrange rule. This result is somewhat motivated by the ideas in [14, Theorem 3.1]. The proof of [21, Theorem 2.2] follows similar lines but in a different, nonhomogeneous context. The local boundedness of nontrivial solutions has been addressed in [5] as well. Our results are closely related to the study of the asymptotic properties of particular subsequences of eigenvalues. The characterization of these subsequences is an interesting problem which has been raised in several references in the literature and in various settings, such as the classical $p$-Laplacian one and the case of nonhomogeneous variable exponents, see $[22,23]$.

In this manuscript we denote by

$$
\Phi(s)=\int_{0}^{s} \phi(t) d t
$$

the $N$-function induced by $\phi$. (See [1] for a thorough survey on $N$-functions). We assume that there exist positive constants $p_{\Phi}$ and $q_{\Phi}$ such that

$$
1<p_{\Phi} \leq \frac{t \phi(t)}{\Phi(t)} \leq q_{\Phi}<+\infty, \text { for } t \neq 0 .
$$

We then formulate the following eigenvalue problem

$$
-\Delta_{\phi} u=\lambda g(\cdot) \phi(u) \text { in } \mathbb{R}^{N}
$$

on a homogeneous Orlicz space, where $g$ is a real measurable function and $N \geq$ 2 is an integer. If additional, mild restrictions are imposed on $g$, we prove that solutions of this equation are regular, positive and vanish at infinity through an instrumental result (Theorem 3.1). The latter (which is consequence of an extended Harnack's inequality proved in [2]) states that the oscillation of the solutions is locally bounded by the supremum of the function on a ball. We stress the fact that $\phi$ need not be differentiable. (Compare our fundamental estimates (1.2) with bounds (1.1) in [20]).

As a final remark, we mention that the results presented in these notes generalize those of J. Fleckinger, R. Manásevich, N. Stavrakakis and F. De Thélin [8]. In that reference the authors treat problem (1.3) in the particular case of the $p$-Laplacian operator for which $\phi(s)=|s|^{p-2} s, p>1$. The authors 
use a priori estimates from [24] to determine the regularity as well as the asymptotic behavior of the solutions. Positivity of eigenfunctions is proved via Vasquez's Maximum Principle [25].

\section{Generalities}

The $N$-function (1.1) associated to the odd increasing homeomorphism $\phi: \mathbb{R} \rightarrow \mathbb{R}$ is even, strictly increasing, convex and continuous. Note that integration of the inequality on the right in (1.2) yields for $\kappa>1$ and $t>0$ the estimate $\Phi(\kappa t) \leq \kappa^{q_{\Phi}} \Phi(t)$. Therefore,

$$
\phi(\kappa t) \leq q_{\Phi} \frac{\Phi(\kappa t)}{\kappa t} \leq q_{\Phi} \kappa^{q_{\Phi}-1} \frac{\Phi(t)}{t} \leq q_{\Phi} \kappa^{q_{\Phi}-1} \phi(t)
$$

The conjugate (or complementary) $N$-function $\bar{\Phi}$ of $\Phi$ is defined by

$$
\bar{\Phi}(t)=\int_{0}^{t} \phi^{-1}(s) d s
$$

where $\phi^{-1}$ is the inverse of $\phi$. Young's inequality [17] and (1.2) imply

$$
\bar{\Phi}(\phi(t)) \leq t \phi(t) \leq q_{\Phi} \Phi(t) \text { for all } t \in \mathbb{R} .
$$

The Sobolev conjugate $N$-function $\Phi_{*}$ of $\Phi$ is defined via

$$
\Phi_{*}^{-1}(t)=\int_{0}^{t} \frac{\Phi^{-1}(s)}{s^{(N+1) / N}} d s
$$

where $\Phi^{-1}$ is the inverse of $\left.\Phi\right|_{[0, \infty)}$. This definition assumes that $\Phi_{*}^{-1}(1)$ exists and $\lim _{t \rightarrow+\infty} \Phi_{*}^{-1}(t)=+\infty$.

The Sobolev conjugate exponents $p_{\Phi}^{*}=N p_{\Phi} /\left(N-p_{\Phi}\right)$ and $q_{\Phi}^{*}=N q_{\Phi} /(N-$ $\left.q_{\Phi}\right)$ are well-defined provided $q_{\Phi}<N$. It is evident that $1<p_{\Phi}^{*} \leq q_{\Phi}^{*}$.

Lemma 2.1. [9] Let $\rho, t$ be nonnegative real numbers. If $q_{\Phi}<N$ then
a) $\min \left\{\rho^{p_{\Phi}}, \rho^{q_{\Phi}}\right\} \Phi(t) \leq \Phi(\rho t) \leq \max \left\{\rho^{p_{\Phi}}, \rho^{q_{\Phi}}\right\} \Phi(t)$;
b) $\min \left\{\rho^{p_{\Phi}^{*}}, \rho^{q_{\Phi}^{*}}\right\} \Phi_{*}(t) \leq \Phi_{*}(\rho t) \leq \max \left\{\rho^{p_{\Phi}^{*}}, \rho^{q_{\Phi}^{*}}\right\} \Phi_{*}(t)$;
c) $\min \left\{\rho^{p_{\Phi}^{*} /\left(p_{\Phi}^{*}-1\right)}, \rho^{q_{\Phi}^{*} /\left(q_{\Phi}^{*}-1\right)}\right\} \overline{\Phi_{*}}(t) \leq \overline{\Phi_{*}}(\rho t) \leq \max \left\{\rho^{p_{\Phi}^{*} /\left(p_{\Phi}^{*}-1\right)}, \rho^{q_{\Phi}^{*} /\left(q_{\Phi}^{*}-1\right)}\right\} \overline{\Phi_{*}}(t)$.

The $\Delta_{2}$-condition. It is known [1] that fundamental estimates (1.2) ensure that $\Phi$ satisfies a global $\Delta_{2}$-condition. This means that there exists a positive constant $C^{\prime}$ such that for every $t \geq 0, \Phi(2 t) \leq C^{\prime} \Phi(t)$. By Lemma 2.5 in [9] the hypothesis $p_{\Phi}>1$ implies that the complementary $N$-function $\bar{\Phi}$ satisfies a $\Delta_{2}$-condition as well. Lemma 2.1 ensures that the Sobolev conjugate $\Phi_{*}$ and its complementary $\overline{\Phi_{*}}$ also satisfy a $\Delta_{2}$-condition provided $q_{\Phi}<N$. 


\subsection{Orlicz-Sobolev spaces}

This section is a brief survey on Orlicz and Orlicz-Sobolev spaces. We let $\Omega \subseteq \mathbb{R}^{N}$ be an open domain and denote by $\mathcal{D}(\Omega)$ the space of infinitelydifferentiable functions with compact support in $\Omega$. We let $\Phi$ be the $N$-function from the previous section, induced by the homeomorphism $\phi: \mathbb{R} \rightarrow \mathbb{R}$ via (1.1). The Orlicz class $\mathcal{L}_{\Phi}(\Omega)$ is the set of (equivalence classes of) real-valued measurable functions $u$ such that $\Phi(u) \in L^{1}(\Omega)$. In general, $\mathcal{L}_{\Phi}(\Omega)$ is not a vector space [11]. The linear hull $L_{\Phi}(\Omega)$ of $\mathcal{L}_{\Phi}(\Omega)$ is a vector space which is Banach with the Luxemburg norm

$$
\|u\|_{\Phi, \Omega}=\inf \left\{k>0: \int_{\Omega} \Phi\left(\frac{u}{k}\right) d x \leq 1\right\} .
$$

The closure in $L_{\Phi}(\Omega)$ of the space of bounded measurable functions with compact support in $\bar{\Omega}$ is denoted by $E_{\Phi}(\Omega)$. This space is separable and Banach with the inherited norm. In general, $E_{\Phi}(\Omega) \subseteq \mathcal{L}_{\Phi}(\Omega) \subseteq L_{\Phi}(\Omega)$. However, it is known that $\Phi$ satisfies a global $\Delta_{2}$-condition if and only if $E_{\Phi}(\Omega)=L_{\Phi}(\Omega)$. Theorem 8.20 in [1] guarantees that the spaces $L_{\Phi}(\Omega)$ and $L_{\bar{\Phi}}(\Omega)$ are reflexive and separable, since $\bar{\Phi}$ also satisfies a $\Delta_{2}$-condition. From $[1,11]$ it follows that one can identify the dual space of $E_{\Phi}(\Omega)$ with $L_{\bar{\Phi}}(\Omega)$ and the dual space of $E_{\bar{\Phi}}(\Omega)$ with $L_{\Phi}(\Omega)$. It is also known that if $u \in L_{\Phi}(\Omega)$ and $v \in L_{\bar{\Phi}}(\Omega)$, then

$$
\int_{\Omega}|u v| d x \leq 2\|u\|_{\Phi, \Omega}\|v\|_{\bar{\Phi}, \Omega}
$$

which is an extension of Hölder's inequality to Orlicz spaces.

In the case $\Omega=\mathbb{R}^{N}$ we define the homogeneous Orlicz space $\mathscr{D}_{\mathrm{O}}^{1, \Phi}\left(\mathbb{R}^{N}\right)$ to be the closure of $\mathcal{D}\left(\mathbb{R}^{N}\right)$ with respect to the norm

$$
\|u\|=\|u\|_{\Phi_{*}, \mathbb{R}^{N}}+\|\nabla u\|_{\Phi, \mathbb{R}^{N}}
$$

where $\|\nabla u\|_{\Phi, \mathbb{R}^{N}}=\sum_{i=1}^{N}\left\|\partial_{x_{i}} u\right\|_{\Phi, \mathbb{R}^{N}}$. By [7, Theorem 3.4], if $u \in \mathcal{D}\left(\mathbb{R}^{N}\right)$ then $\|u\|_{\Phi_{*}, \mathbb{R}^{N}} \leq \mathscr{C}(N)\|\nabla u\|_{\Phi, \mathbb{R}^{N}}$ where $\mathscr{C}(N)$ is a positive constant. This inequality extends to all of $\mathscr{D}_{0}^{1, \Phi}\left(\mathbb{R}^{N}\right)$ by density and then the norms

$$
\|\cdot\|_{\mathscr{D}_{\mathrm{O}}^{1, \Phi}\left(\mathbb{R}^{N}\right)}=\|\nabla(\cdot)\|_{\Phi, \mathbb{R}^{N}}
$$

and (2.4) are equivalent on $\mathscr{D}_{\mathrm{o}}^{1, \Phi}\left(\mathbb{R}^{N}\right)$. Moreover, by definition of the distributional derivative and by Hölder's inequality (2.3), the set

$$
\mathscr{D}^{1, \Phi}\left(\mathbb{R}^{N}\right)=\left\{u \in L_{\Phi_{*}}\left(\mathbb{R}^{N}\right):|\nabla u| \in L_{\Phi}\left(\mathbb{R}^{N}\right)\right\}
$$

is a Banach space with respect to the norm $(2.4)$ and since $\mathcal{D}\left(\mathbb{R}^{N}\right) \subseteq \mathscr{D}^{1, \Phi}\left(\mathbb{R}^{N}\right)$,

$$
\mathscr{D}_{\mathrm{O}}^{1, \Phi}\left(\mathbb{R}^{N}\right) \subseteq \mathscr{D}^{1, \Phi}\left(\mathbb{R}^{N}\right)
$$


A thorough study of the properties of the homogeneous Orlicz space $\mathscr{D}_{\mathrm{o}}^{1, \Phi}\left(\mathbb{R}^{N}\right)$ has been recently published, see [4].

Orlicz-Sobolev spaces. The Orlicz-Sobolev space $W^{1} L_{\Phi}(\Omega)$ (respectively $W^{1} E_{\Phi}(\Omega)$ ) is the vector subspace of functions in $L_{\Phi}(\Omega)$ (respectively $E_{\Phi}(\Omega)$ ) with first distributional derivatives in $L_{\Phi}(\Omega)$ (respectively $E_{\Phi}(\Omega)$ ). These spaces are Banach with the norm

$$
\|u\|_{1, \Phi, \Omega}=\|u\|_{\Phi, \Omega}+\|\nabla u\|_{\Phi, \Omega}
$$

where $\|\nabla u\|_{\Phi, \Omega}=\sum_{i=1}^{N}\left\|\partial_{x_{i}} u\right\|_{\Phi, \Omega}$. Usually, $W^{1} L_{\Phi}(\Omega)$ and $W^{1} E_{\Phi}(\Omega)$ are identified with subspaces of the products $\Pi L_{\Phi}(\Omega)$ and $\Pi E_{\Phi}(\Omega)$, respectively. The natural embedding of $W^{1} E_{\Phi}(\Omega)$ into the product proves that $W^{1} E_{\Phi}(\Omega)$ is separable since $E_{\Phi}(\Omega)$ is itself separable. The space $W^{1} L_{\Phi}(\Omega)$ is not separable in general. The Orlicz-Sobolev subspaces $W_{0}^{1} L_{\Phi}(\Omega)$ and $W_{0}^{1} E_{\Phi}(\Omega)$ are defined to be the $\sigma$-closure (where $\sigma$ denotes the weak-* topology $\sigma\left(\Pi L_{\Phi}, \Pi E_{\bar{\Phi}}\right)$ ) and the norm-closure, respectively, of the space $\mathcal{D}(\Omega)$ in $W^{1} L_{\Phi}(\Omega)$ :

$$
W_{0}^{1} L_{\Phi}(\Omega)=\overline{\mathcal{D}(\Omega)}^{\sigma} \text { and } W_{0}^{1} E_{\Phi}(\Omega)=\overline{\mathcal{D}(\Omega)}^{\|\cdot\|_{1, \Phi, \Omega}} .
$$

The space $W_{0}^{1} L_{\Phi}(\Omega)$ is Banach for the norm inherited from $W^{1} L_{\Phi}(\Omega)$.

Definition 2.1. The domain $\Omega$ has the segment property if every $x \in \partial \Omega$ has a neighborhood $\mathcal{U}_{x}$ and a non-zero vector $y_{x} \in \mathbb{R}^{N}$ such that if $z \in \bar{\Omega} \cap \mathcal{U}_{x}$, then $z+t y_{x} \in \Omega$, for $0<t<1$.

It is known [11] that if $\Omega$ has the segment property then $W_{0}^{1} E_{\Phi}(\Omega)=$ $W_{0}^{1} L_{\Phi}(\Omega) \cap \Pi E_{\Phi}(\Omega)$. It is evident that if $\Phi$ satisfies a $\Delta_{2}$-condition then $\mathcal{D}(\Omega)$ is dense in $W_{0}^{1} L_{\Phi}(\Omega)$ for the norm $\|\cdot\|_{1, \Phi, \Omega}$ inherited from $W^{1} L_{\Phi}(\Omega)$.

For further details on Orlicz and Orlicz-Sobolev spaces we refer the reader to $[1,11,17]$. Spaces defined by variable exponents $p(x)$, where $p: \Omega \rightarrow$ $(1,+\infty)$ is a bounded function, are treated in [22] and [23]. These articles study the case of nonhomogeneous differential operators containing one or more variable power-type nonlinearities. The theory there is developed in great generality including many possible pathologies of the underlying $N$ function. In addition, this variable formulation leads to the study of conditions for removability of isolated singular points of elliptic equations in the Sobolev space $W^{1, p(x)}(\Omega)$, which was first studied by Kováčik and Rákosník [16].

\subsection{The property $(\mathscr{P})$.}

Let $\Omega$ be an open and bounded domain in $\mathbb{R}^{N}$. Let $\mathcal{B}: \Omega \times W^{1} L_{\Phi}(\Omega) \rightarrow \mathbb{R}$ be a Carathéodory function fulfilling the growth condition

$$
|\mathcal{B}(x, u)| \leq \mathfrak{a} \phi(|u(x)|)+\mathfrak{b} \quad \text { a.e. } x \text { in } \Omega
$$


where $\mathfrak{a}, \mathfrak{b}$ are two fixed, nonnegative real numbers. We will say that a function $u \in W^{1} L_{\Phi}(\Omega)$ satisfies the property $(\mathscr{P})$ in the domain $\Omega$ if

$$
\int_{\Omega} \phi(|\nabla u|) \frac{\nabla u}{|\nabla u|} \cdot \nabla \theta d x=\int_{\Omega} \mathcal{B}(x, u) \theta d x
$$

for all $\theta \in W_{0}^{1} L_{\Phi}(\Omega)$. The validity of this variational formulation is justified as follows. Since $\Phi$ satisfies a $\Delta_{2}$-condition, $L_{\Phi}(\Omega)=\mathcal{L}_{\Phi}(\Omega)$. Hence, bounds (2.2) yield $\phi(|\nabla u|) \in L_{\bar{\Phi}}(\Omega)$ for any $u \in W^{1} L_{\Phi}(\Omega)$. Since $\theta \in W^{1} L_{\Phi}(\Omega)$, by definition we have that both $\theta$ and $\nabla \theta$ belong to $L_{\Phi}(\Omega)$. Hölder's extended inequality (2.3) implies that the integral on the left is finite. Estimate $(2.2)$ also yields $\phi(u) \in L_{\bar{\Phi}}(\Omega)$. Since $\Omega$ has finite volume, bound (2.6) implies that the right-hand side is finite as well.

Let $u$ be a bounded and nonnegative function satisfying property $(\mathscr{P})$ in $\Omega$. Let $B_{R} \subset \subset \Omega$ denote a ball of radius $0<R \leq 1$ and $B_{R / 2}$ be the ball of radius $R / 2$ concentric with $B_{R}$. Choose any real nonnegative number $L$ such that $\mathfrak{b} \leq \phi(L)$. The following average estimate is proved in [2] for any $p>0$ :

$$
\sup _{B_{R / 2}} u \leq \mathcal{M}\left(\left(\int_{B_{R}} u^{p} d x\right)^{1 / p}+L R\right)
$$

where $\mathcal{M}=\mathcal{M}\left(\mathfrak{a}, p, q_{\Phi}, N\right)$ is a positive constant and $f$ denotes the average (mean) integral of $u^{p}$ on the ball $B_{R}$. This bound is indeed an intermediate result in the proof of the extended Harnack's inequality

$$
\sup _{B_{R / 2}} u \leq \mathcal{N}\left(\inf _{B_{R / 2}} u+L R\right)
$$

where $\mathcal{N}=\mathcal{N}\left(\mathfrak{a}, p_{\Phi}, q_{\Phi}, N\right)$ is a positive constant [2, Theorem 1.1]

\section{Oscillation of solutions}

In this section we study the oscillation of nontrivial and nonnegative solutions of problem (1.3), see below. This result will be instrumental in the determination of the regularity and asymptotic properties of these functions.

In the sequel, and in addition to fundamental estimates (1.2), we assume the following hypotheses:

$\left(\mathcal{H}_{1}\right) q_{\Phi}<N$ and $q_{\Phi}<p_{\Phi}^{*}$

$\left(\mathcal{H}_{2}\right) g \in L^{q_{\Phi}^{*} /\left(q_{\Phi}^{*}-p_{\Phi}\right)}\left(\mathbb{R}^{N}\right) \cap L^{\infty}\left(\mathbb{R}^{N}\right)$. 
Hypothesis $\left(\mathcal{H}_{2}\right)$ generalizes the natural condition $g \in L^{N / p}\left(\mathbb{R}^{N}\right) \cap L^{\infty}\left(\mathbb{R}^{N}\right)$ imposed on the right-hand side of the $p$-Laplacian eigenvalue differential problem considered in [8]. Lemma 2.1 and hypotheses $\left(\mathcal{H}_{1}\right)$ and $\left(\mathcal{H}_{2}\right)$ imply the following result.

Corollary 3.1. [5] If $u \in L_{\Phi_{*}}\left(\mathbb{R}^{N}\right)$ then $g \phi(u) \in L_{\overline{\Phi_{*}}}\left(\mathbb{R}^{N}\right)$.

Therefore, if $u, \theta \in L_{\Phi_{*}}\left(\mathbb{R}^{N}\right)$ then Hölder's inequality (2.3) produces

$$
\int_{\mathbb{R}^{N}} g(x) \phi(u) \theta d x<+\infty .
$$

This motivates the following definition.

Definition 3.1. A function $u \in \mathscr{D}_{\mathrm{o}}^{1, \Phi}\left(\mathbb{R}^{N}\right)$ is a solution of $(1.3)$ if there exists $\lambda \in \mathbb{R}$ such that

$$
\int_{\mathbb{R}^{N}} \phi(|\nabla u|) \frac{\nabla u}{|\nabla u|} \cdot \nabla \theta d x=\lambda \int_{\mathbb{R}^{N}} g(x) \phi(u) \theta d x
$$

for all $\theta \in \mathscr{D}_{\mathrm{o}}^{1, \Phi}\left(\mathbb{R}^{N}\right)$. The number $\lambda$ is called an eigenvalue of (1.3) with associated eigenfunction $u$.

Notice that (2.2) now yields $\bar{\Phi}(\phi(|\nabla u|)) \leq q_{\Phi} \Phi(\nabla u) \in L^{1}\left(\mathbb{R}^{N}\right)$. Since $\Phi$ satisfies a $\Delta_{2}$-condition the left-hand side is finite as well. The connection between solutions of (1.3) and functions satisfying property $(\mathscr{P})$ in any bounded subdomain $\Omega \subseteq \mathbb{R}^{N}$ which has the segment property is given in the next proposition.

Proposition 3.1. Any nonnegative solution $u$ of problem (1.3) fulfills

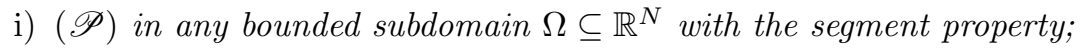

ii) condition (2.6) with $\mathfrak{a}=|\lambda|\|g\|_{\infty}$ and $\mathfrak{b}=0$.

Proof. Let $u$ be a nonnegative solution of (1.3). In particular, $\phi(|\nabla u|) \in$ $L_{\bar{\Phi}}(\Omega)$ for any subdomain $\Omega \subseteq \mathbb{R}^{N}$. Likewise, inclusion (2.5) implies $u \in$ $\mathscr{D}^{1, \Phi}\left(\mathbb{R}^{N}\right)$ and then $u \in L_{\Phi_{*}}(\bar{\Omega})$ and $|\nabla u| \in L_{\Phi}(\Omega)$ on the same domain. Proposition 2.1 in [10] says that $\Phi$ increases essentially more slowly than $\Phi_{*}$ near infinity [1, pp. 265]. Theorem 8.16 in [1] ensures that $L_{\Phi_{*}}(\Omega)$ is embedded continuously in $E_{\Phi}(\Omega)=L_{\Phi}(\Omega)$ (the latter equality holds since $\Phi$ satisfies a $\Delta_{2}$-condition) and thus $u \in W^{1} L_{\Phi}(\Omega)$.

Suppose that the domain $\Omega$ has the segment property. The remark after Definition 2.1 implies that we can approximate any given $\theta \in W_{0}^{1} L_{\Phi}(\Omega)$ by a sequence $\left\{\theta_{n}\right\} \in \mathcal{D}(\Omega)$ with respect to the norm inherited from $W^{1} L_{\Phi}(\Omega)$. 
Let $\bar{\theta}_{n}$ denote the extension of $\theta_{n}$ by zero outside $\Omega$. It follows that $\left\{\bar{\theta}_{n}\right\} \subseteq$ $\mathcal{D}\left(\mathbb{R}^{N}\right) \subseteq \mathscr{D}_{\mathrm{o}}^{1, \Phi}\left(\mathbb{R}^{N}\right)$. Since $\bar{\theta}_{n} \equiv 0$ in $\Omega^{c}$ the variational formula (3.1) yields

$$
\int_{\Omega} \phi(|\nabla u|) \frac{\nabla u}{|\nabla u|} \cdot \nabla \theta_{n} d x=\lambda \int_{\Omega} g(x) \phi(u) \theta_{n} d x \text { for all } n \in \mathbb{N} .
$$

Since $\bar{\Phi}$ satisfies a $\Delta_{2}$-condition, estimate (2.2) produces again $\bar{\Phi}(g(x) \phi(u)) \leq$ $C q_{\Phi} \Phi(u) \in L^{1}(\Omega)$ for a positive constant $C$. Hölder's inequality enables us to pass to the limit $n \rightarrow+\infty$ in the variational formula above and thus this equivalence is valid with $\theta_{n}$ replaced by $\theta$. Then the solution $u$ satisfies property $(\mathscr{P})$ with the right-hand side $\mathcal{B}(x, u)=\lambda g(x) \phi(u)$. The proof of ii) is thus immediate.

Lemma 3.1. [6] Let $\tau$ be a non-decreasing function and $\rho, \beta \in(0,1)$ such that $\tau(\rho r) \leq \beta \tau(r)+g(r)$, where $0<r \leq R$ and $g$ is also non-decreasing. Then

$$
\tau(r) \leq \frac{1}{\beta}\left(\frac{r}{R}\right)^{(1-\mu) \frac{\log \beta}{\log \rho}} \tau(R)+\frac{g\left(R^{1-\mu} r^{\mu}\right)}{1-\beta}
$$

where $\mu \in(0,1)$ and $0<r \leq R$.

Theorem 3.1. Let $u$ be a solution of equation (1.3) with associated eigenvalue $\lambda$. Let $B_{r} \subseteq B_{R}$ be two concentric balls in $\Omega$ of radii $0<r \leq R \leq 1$, respectively. Then there exists a constant $\gamma \in(0,1)$ such that the oscillation

$$
\underset{B_{r}}{\operatorname{OSc}} u=\sup _{B_{r}} u-\inf _{B_{r}} u \leq \mathfrak{C}\left(\frac{r}{R}\right)^{\gamma}\left(\sup _{B_{R}}|u|+L R\right)
$$

where $\mathfrak{C}=\mathfrak{C}\left(\lambda,\|g\|_{\infty}, p_{\Phi}, q_{\Phi}, N\right)>0$ and $L$ is any positive constant such that

$$
|\lambda|\|g\|_{\infty} 2^{q_{\Phi}-1} q_{\Phi}^{2} \phi\left(\|u\|_{\infty, B_{R}}\right) \leq \phi(L) .
$$

The term $\|u\|_{\infty, B_{R}}$ denotes the norm of the essential supremum (resp. maximum) of $u$ on $B_{R}$.

Proof. Define $\chi=\chi(r)=\sup _{B_{r}} u$ and $\theta=\theta(r)=\inf _{B_{r}} u$. Consider the (nonnegative) function $v=u-\theta(r)$ in $B_{r}$. By Proposition 3.1, $v$ satisfies $\operatorname{property}(\mathscr{P})$ in $B_{r}$ with $\mathcal{B}(x, v)=\lambda g(x) \phi(v+\theta)$. Hence

Take $\alpha, \beta \geq 0$ such that either $\alpha$ or $\beta$ is strictly positive so that $\alpha+\beta>0$.

$$
\begin{aligned}
(\alpha+\beta) \phi(\alpha+\beta) \leq q_{\Phi} \Phi(\alpha+\beta) & \leq q_{\Phi}(\Phi(2 \alpha)+\Phi(2 \beta)) / 2 \\
& \leq q_{\Phi}(\alpha \phi(2 \alpha)+\beta \phi(2 \beta)) \\
& \leq q_{\Phi}(\alpha+\beta)(\phi(2 \alpha)+\phi(2 \beta))
\end{aligned}
$$


and then $\phi(\alpha+\beta) \leq q_{\Phi}(\phi(2 \alpha)+\phi(2 \beta))$. Inequality (2.1) implies $\phi(2 \alpha) \leq$ $q_{\Phi} 2^{q_{\Phi}-1} \phi(\alpha)$ and $\phi(2 \beta) \leq q_{\Phi} 2^{q_{\Phi}-1} \phi(\beta)$. Thus

$$
\phi(\alpha+\beta) \leq 2^{q_{\Phi}-1} q_{\Phi}^{2}(\phi(\alpha)+\phi(\beta)) .
$$

Note that if $\alpha=\beta=0$ then this inequality holds as well. This estimate yields

$$
|\lambda g(x) \phi(v+\theta)| \leq \mathfrak{a} \phi(v)+\mathfrak{b}
$$

where $\mathfrak{a}=|\lambda|\|g\|_{\infty} 2^{q_{\Phi}-1} q_{\Phi}^{2}$ and $\mathfrak{b}=|\lambda|\|g\|_{\infty} 2^{q_{\Phi}-1} q_{\Phi}^{2} \phi\left(\|u\|_{\infty, B_{R}}\right)$. Harnack's inequality (2.8) implies

$$
\chi(r / 2)-\theta(r)=\sup _{B_{r / 2}} v \leq \mathcal{N}\left(\inf _{B_{r / 2}} v+L r\right)=\mathcal{N}(\theta(r / 2)-\theta(r)+L r)
$$

where $0<r \leq R, \mathcal{N}=\mathcal{N}\left(\lambda,\|g\|_{\infty}, p_{\Phi}, q_{\Phi}, N\right)$ and $L$ is any positive constant such that $\mathfrak{b} \leq \phi(L)$.

On the other hand, the function $w=\chi(r)-u$ satisfies property $(\mathscr{P})$ in $B_{r}$ with $\mathcal{B}(x, w)=-\lambda g(x) \phi(\chi-w)$. Hence

$$
\chi(r)-\theta(r / 2)=\sup _{B_{r / 2}} w \leq \overline{\mathcal{N}}\left(\inf _{B_{r / 2}} w+L r\right)=\overline{\mathcal{N}}(\chi(r)-\chi(r / 2)+L r) .
$$

where $\overline{\mathcal{N}}=\overline{\mathcal{N}}\left(\lambda,\|g\|_{\infty}, p_{\Phi}, q_{\Phi}, N\right)$ as well and $L$ is the same constant above. Taking $C=\max \{\mathcal{N}, \overline{\mathcal{N}}\}$ and adding (3.2) and (3.3) produces

$$
\chi(r / 2)-\theta(r / 2) \leq \frac{C-1}{C+1}(\chi(r)-\theta(r))+\frac{2 C L r}{C+1} .
$$

That is,

$$
\tau(r / 2) \leq \frac{C-1}{C+1} \tau(r)+\frac{2 C L r}{C+1}
$$

where $\tau(r)=\operatorname{osc}_{B_{r}} u=\chi(r)-\theta(r)$. Lemma 3.1 with $\gamma=(1-\mu) \log ((C-$ 1) $/(C+1)) / \log (1 / 2), 0<r \leq R$ and $0<\mu<1$ implies

$$
\tau(r) \leq \frac{C+1}{C-1}\left(\frac{r}{R}\right)^{\gamma} \tau(R)+C L R^{1-\mu} r^{\mu} .
$$

Choose $\mathfrak{C}=\max \{2(C+1) /(C-1), C\}$ and $\gamma<\mu$, i.e. $\mu$ near to 1 . Since $\tau(R) \leq 2 \sup _{B_{R}}|u|$, the result is proved. 


\section{Regularity, strict positivity and vanishing at infinity}

Let $\Omega$ be an open domain in $\mathbb{R}^{N}$. Recall that a function $u: \Omega \mapsto \mathbb{R}$ is uniformly Hölder continuous with exponent $0<\gamma \leq 1$ if the quantity

$$
[u]_{\gamma, \Omega}=\sup _{\substack{x, y \in \Omega \\ x \neq y}} \frac{|u(x)-u(y)|}{|x-y|^{\gamma}}
$$

is finite. (Since this number is zero on constant functions, it only defines a semi-norm). The function $u$ is locally uniformly Hölder continuous with exponent $\gamma$ in $\Omega$ if the quantity $[u]_{\gamma, \Omega^{\prime}}$ is finite for every $\Omega^{\prime} \subset \subset \Omega$. We denote by $C^{0, \gamma}(\Omega)$ the space of locally uniformly Hölder continuous functions with exponent $\gamma$ in $\Omega$. If $\Omega$ is bounded this space is denoted by $C^{0, \gamma}(\bar{\Omega})$. It is known that the latter is Banach with respect to the norm

$$
\|u\|_{C^{\gamma}(\bar{\Omega})}=[u]_{\gamma, \Omega}+\|u\|_{\infty, \Omega}
$$

where $\|\cdot\|_{\infty, \Omega}$ is the norm of the essential supremum (resp. maximum) on $\Omega$.

Lemma 4.1. [5] Let $u$ be a solution of equation (1.3) with associated eigenvalue $\lambda$ and let $0<R \leq 1$. Then there exists a positive constant $\mathbf{C}=$ $\mathbf{C}\left(\lambda,\|g\|_{\infty}, q_{\Phi}, N\right)$ such that

$$
\|\Phi(|u|)\|_{L^{\infty}\left(B_{R / 2}\right)} \leq \frac{\mathbf{C}}{R^{N q_{\Phi}}}\|\Phi(|u|)\|_{L^{1}\left(B_{R}\right)}
$$

where $B_{R / 2}$ is the ball of radius $R / 2$ concentric with $B_{R}$.

Theorem 4.1. Let $u$ be a solution of equation (1.3) with associated eigenvalue $\lambda$. Let $\Omega$ be a bounded domain in $\mathbb{R}^{N}$. Then for any subdomain $\Omega_{0} \subset \subset \Omega$ there exist constants $\gamma \in(0,1)$ and $\mathcal{L}>0$ such that

$$
\|u\|_{C^{\gamma}\left(\overline{\Omega_{0}}\right)} \leq \mathfrak{m}\left(\|u\|_{L^{1}(\Omega)}+\mathcal{L}\right)
$$

where $\mathfrak{m}=\mathfrak{m}\left(\lambda,\|g\|_{\infty}, \gamma, d, p_{\Phi}, q_{\Phi}, N\right)$ is positive and $d=\operatorname{dist}\left(\Omega_{0}, \partial \Omega\right)$.

Proof. Let $R<\operatorname{dist}\left(\partial \Omega, \Omega_{0}\right) / 8$ and $\theta=q_{\Phi} / \phi(1)$. The adherence $\overline{\Omega_{0}}$ is compact and then $\Omega_{0}$ can be covered by finitely many balls $B_{R / 4}\left(x_{j}\right)$ where $x_{j} \in \overline{\Omega_{0}}$ and $j=1, \ldots, M$ with $M=M\left(R,\left|\Omega_{0}\right|\right)$ a positive integer. Since $B_{R / 2}\left(x_{j}\right) \subseteq \Omega$, inequality $t \leq \theta \Phi(t)+1(t \geq 0)$ and Lemma 4.1 imply

$$
\begin{aligned}
\sup _{\Omega_{0}}|u| \leq \max _{j} \sup _{B_{R / 4}\left(x_{j}\right)}|u| & \left.\leq \max _{j}\left(\frac{\theta \mathbf{C}}{R^{N q_{\Phi}}}\|\Phi(u)\|_{L^{1}\left(B_{R / 2}\right)}\left(x_{j}\right)\right)+1\right) \\
& \leq \frac{\theta \mathbf{C}}{R^{N q_{\Phi}}}\|\Phi(u)\|_{L^{1}(\Omega)}+1
\end{aligned}
$$

where $\mathbf{C}=\mathbf{C}\left(\lambda,\|g\|_{\infty}, q_{\Phi}, N\right)$. Let us fix $x, y \in \Omega_{0}$. Two different cases arise. 
First case. Suppose that the absolute value $r=|x-y| \leq R$. Theorem 3.1 (with $2 R \leq 1$ ) along with Lemma 4.1 yield

$$
\begin{aligned}
\underset{B_{r}(x)}{\operatorname{Osc}} u & \leq \mathfrak{C}\left(\frac{r}{2 R}\right)^{\gamma}\left(\sup _{B_{2 R}(x)}|u|+2 L R\right) \\
& \leq \mathfrak{C} r^{\gamma}\left(2^{-\gamma} \theta \mathbf{C} R^{-\gamma-N q_{\Phi}}\|\Phi(u)\|_{L^{1}\left(B_{4 R}(x)\right)}+(2 R)^{-\gamma}(1+2 L R)\right) \\
& \leq \mathfrak{C} r^{\gamma}\left(2^{-\gamma} \theta \mathbf{C} R^{-\gamma-N q_{\Phi}}\|\Phi(u)\|_{L^{1}(\Omega)}+(2 R)^{-\gamma}(1+2 L R)\right) .
\end{aligned}
$$

Then

$$
|u(x)-u(y)| \leq \underset{B_{r}(x)}{\operatorname{Osc}} u \leq C|x-y|^{\gamma}\left\{\|\Phi(u)\|_{L^{1}(\Omega)}+\kappa\right\}
$$

where $C=C\left(\lambda,\|g\|_{\infty}, \gamma, p_{\Phi}, q_{\Phi}, N, R\right)$ and $\kappa$ are positive constants. The claimed estimate thus follows.

Second case. The number $r=|x-y|>R$. In this case we consider a chain of balls $\left\{B_{R / 4}\left(p_{1}\right), \ldots, B_{R / 4}\left(p_{k}\right)\right\}$, where $k \leq M$ such that $x \in B_{R / 4}\left(p_{1}\right), y \in$ $B_{R / 4}\left(p_{k}\right)$ and $B_{R / 4}\left(p_{j}\right) \cap B_{R / 4}\left(p_{j+1}\right) \neq \varnothing$ for all $j=1, \ldots, k-1$. The triangle inequality and first case above imply

$$
\begin{aligned}
|u(x)-u(y)| & \leq\left|u(x)-u\left(p_{1}\right)\right|+\sum_{j=1}^{k-1}\left|u\left(p_{j}\right)-u\left(p_{j+1}\right)\right|+\left|u\left(p_{k}\right)-u(y)\right| \\
& \leq C\left(\left|x-p_{1}\right|^{\gamma}+\sum_{j=1}^{k-1}\left|p_{j}-p_{j+1}\right|^{\gamma}+\left|p_{k}-y\right|^{\gamma}\right)\left\{\|\Phi(u)\|_{L^{1}(\Omega)}+\kappa\right\} \\
& \leq C(k+1)|x-y|^{\gamma}\left\{\|\Phi(u)\|_{L^{1}(\Omega)}+\kappa\right\} \\
& \leq C(M+1)|x-y|^{\gamma}\left\{\|\Phi(u)\|_{L^{1}(\Omega)}+\kappa\right\}
\end{aligned}
$$

where $C=C\left(\lambda,\|g\|_{\infty}, \gamma, p_{\Phi}, q_{\Phi}, N, R\right)$ and $\kappa$ are positive constants. The desired inequality for the Hölder seminorm follows.

Let $u$ be a solution of equation (1.3). Following [15, Corollary 11.1.2], we choose a subdomain $\Omega_{0} \subset \subset \Omega$. Then the adherence $\overline{\Omega_{0}}$ is compact and $\Omega_{0}$ can be covered by finitely many balls $B_{i}=B_{R}\left(x_{i}\right) \subseteq \Omega$ where $R<\operatorname{dist}\left(\partial \Omega, \Omega_{0}\right) / 4$ for all $i=1, \ldots, \ell$. Let $y_{1}, y_{2} \in \Omega_{0}$ such that $y_{1} \in B_{k}$ and $y_{2} \in B_{k+m}$ for some $m \geq 1$ and the balls are enumerated such that $B_{j} \cap B_{j+1} \neq \varnothing$ for $j=k, \ldots k+m-1$. If we apply Harnack's inequality (2.8) on each ball $B_{k}, B_{k+1}, \ldots$ we obtain

$$
u\left(y_{1}\right) \leq \sup _{B_{k}} u \leq \mathcal{N} \inf _{B_{k}} u \leq \cdots \leq \mathcal{N}^{m+1} \inf _{B_{k+m}} u \leq \mathcal{N}^{m+1} u\left(y_{2}\right) .
$$

Write $\mathbf{N}=\mathcal{N}^{\ell+1}$. Since $y_{1}, y_{2}$ are arbitrary and $m \leq \ell$ it follows that

$$
\sup _{\Omega_{0}} u \leq \mathrm{N} \inf _{\Omega_{0}} u .
$$


Theorem 4.2. Any solution $u=u(x)$ of equation (1.3) is strictly positive everywhere in $\mathbb{R}^{N}$ and decays uniformly to zero as $|x| \rightarrow+\infty$.

Proof. Remark 4.3 in [12] applies also in unbounded domains. Hence solutions of (1.3) are nonnegative. Consider the (closed) subset $\Omega^{*}=\left\{x \in \mathbb{R}^{N}: u(x)=\right.$ $0\}$. Let us suppose that $\Omega^{*}$ is non-empty. Then the boundary $\partial \Omega^{*}$ is nonempty so we can take $x_{0} \in \partial \Omega^{*}$ : for any $r>0$ the ball $B_{r}\left(x_{0}\right)$ intersects both $\Omega^{*}$ and $\mathbb{R}^{N} \backslash \Omega^{*}$. Then (4.1) yields

$$
0<\sup _{B_{r}\left(x_{0}\right)} u \leq \mathrm{N} \inf _{B_{r}\left(x_{0}\right)} u=0
$$

which is a contradiction. Thus $u$ is strictly positive in $\mathbb{R}^{N}$.

On the other hand, if $u \in \mathscr{D}_{\mathrm{o}}^{1, \Phi}\left(\mathbb{R}^{N}\right)$ is a solution of (1.3) we have $u \in$ $L_{\Phi_{*}}\left(\mathbb{R}^{N}\right)$. Lemma 2.1 implies that $u \in L^{p_{\Phi}^{*}}\left(\mathbb{R}^{N}\right)$ if $u \geq 1$ and $u \in L^{q_{\Phi}^{*}}\left(\mathbb{R}^{N}\right)$ provided $0<u<1$. Since

$$
\sup _{B_{1 / 2}(z)} u \leq \sup _{B_{1 / 2}(z) \cap\{u \geq 1\}} u+\sup _{B_{1 / 2}(z) \cap\{0<u<1\}} u
$$

inequality (2.7) (applied respectively with $p=p_{\Phi}^{*}, p=q_{\Phi}^{*}$ and $L=0$ ) implies, up to multiplicative factor,

$$
\sup _{B_{1 / 2}(z)} u \leq\left(\int_{B_{1}(z)} u^{p_{\Phi}^{*}} d x\right)^{1 / p_{\Phi}^{*}}+\left(\int_{B_{1}(z)} u^{q_{\Phi}^{*}} d x\right)^{1 / q_{\Phi}^{*}} \rightarrow 0 \quad \text { as } \quad|z| \rightarrow+\infty
$$

where $B_{1 / 2}(z)$ and $B_{1}(z)$ are balls centered at $z \in \mathbb{R}^{N}$ and of radii $1 / 2$ and 1 , respectively. The theorem is proved.

\section{Acknowledgments}

The authors are grateful to Raúl Manásevich (University of Chile) for helpful discussions. The authors also express their gratitude to the referee, who made important remarks and suggested adding some representative items to the list of references.

\section{References}

[1] R.A. Adams and J.F. Fournier, Sobolev Spaces, 2nd edition, Academic Press, 2003.

[2] W. Arriagada and J. Huentutripay. A Harnack's inequality in OrliczSobolev spaces, to appear in Studia Mathematica, (2017). 
[3] W. Arriagada and J. Huentutripay. Blow-up rates of large solutions for a $\phi$-Laplacian problem with gradient term, Proc. A Royal Soc. Edinburgh, 144, No. 1, (2014) 669-689.

[4] W. Arriagada and J. Huentutripay. Characterization of a homogeneous Orlicz space, Electron. J. Differential Equations, Vol. 2017 (2017), No. 49, 1-17.

[5] W. Arriagada and J. Huentutripay. Existence and local boundedness of solutions of a $\phi$-Laplacian problem. To appear in Applicable Analysis, 2017. DOI at http://dx.doi.org/10.1080/00036811.2017.1404581

[6] Ph. Clément and R. Zacher, A priori estimates for weak solutions of elliptic equations, preprint series: 04-30, Reports on Analysis, 2004.

[7] T. Donaldson and N. Trudinger, Orlicz-Sobolev spaces and imbedding theorems, Journal Funct. Anal., 8, (1971), 52-75.

[8] J. Fleckinger, R. Manásevich, N. Stavrakakis and F. De Thélin, Principal Eigenvalues for some Quasilinear Elliptic Equations on $\mathbb{R}^{N}$, Advances in Differential Equations, 2, (1997), 981-1003.

[9] N. Fukagai, M. Ito and K. Narukawa, Positive solutions of Quasilinear Elliptic Equations with Critical Orlicz-Sobolev Nonlinearity on $\mathbb{R}^{N}$, Funkcialaj Ekvacioj, 49, (2006), 235-267.

[10] M. García-Huidobro, V. Le, R. Manásevich and K. Schmitt, On principal eigenvalues for quasilinear elliptic differential operators: An Orlicz-Sobolev space setting, Nonlinear Differential Equations and Applications (NoDEA), 6, (1999), 207-225.

[11] J.-P. Gossez, Orlicz- Sobolev spaces and nonlinear elliptic boundary value problems, Nonlinear Analysis, Function Spaces and Applications. Leipzig: BSB B. G. Teubner Verlagsgesellschaft, (1979), 59-94.

[12] J.-P. Gossez and R. Manásevich, On a nonlinear eigenvalue problem in Orlicz-Sobolev Spaces, Proc. R. Soc. Edinburg, 132A, (2002), 891909.

[13] J. Huentutripay, Estudio de algunas ecuaciones diferenciales de carácter cuasilineal elíptico, PhD thesis, Universidad de Chile, 2009.

[14] J. Huentutripay and R. Manásevich, Nonlinear eigenvalues for a Quasilinear Elliptic System in Orlicz-Sobolev Spaces, Journal of Dynamics and Differential Equations, 18, (2006), 901-929.

[15] J. Jost, Partial differential equations, 3rd ed., Graduate Texts in Mathematics 214, Springer, 2013.

[16] O. Kováčik and J. Rákosník, On spaces $L^{p(x)}$ and $W^{1, p(x)}$, Czechoslovak Math. J., 41, (1991), 592-618. 
[17] M. Krasnosel'skii and J. Rutic'kii, Convex Functions and Orlicz Spaces, Noordhoff, Groningen, 1961.

[18] O.A. Ladyzhenskaya and N.N. Ural'tseva, Linear and Quasilinear Elliptic Equations, Izdat. "Nauka", Moscow, (1964). English transl.: Academic Press, New York, (1968).

[19] O.A. Ladyzhenskaya and N.N. Ural'tseva, Quasilinear elliptic equations and variational problems with many independent variables, Usp. Mat. Nauk, 16 (1961), 19-92. English transl. in Russian Math. Surveys, 16 (1961), 17-91.

[20] G.M. Lieberman, The natural generalization of the natural conditions of Ladyzhenskaya and Ural'tseva for elliptic equations, Comm. In PDE, 16, (1991), 311-361.

[21] M. Mihăilescu, V. Rădulescu and D. Repovš, On a non-homogeneous eigenvalue problem involving a potential: an Orlicz-Sobolev space setting, J. Math. Pures Appl., 93, (2010), 132-148.

[22] V. Rădulescu, Nonlinear elliptic equations with variable exponent: old and new, Nonlinear Analysis: Theory, Methods and Applications, 121, (2015), 336-369.

[23] V. Rădulescu and D. Repovš, Partial Differential Equations with Variable Exponents: Variational Methods and Qualitative Analysis, CRC Press, Taylor \& Francis Group, Boca Raton FL, 2015.

[24] J. Serrin, Local Behavior of Solutions of Quasilinear equations, Acta. Math., 111, (1964), 247-302.

[25] J.L. Vázquez, A Strong Maximum Principle for some Quasilinear Elliptic Equations, Appl. Math. Optim., 12, (1984), 191-202.

Waldo ARRIAGADA,

Department of Applied Mathematics and Sciences,

Khalifa University,

Al Zafranah, P.O. Box 127788, Abu Dhabi, United Arab Emirates.

Email: waldo.arriagada@kustar.ac.ae

Jorge HUENTUTRIPAY

Instituto de Ciencias Físicas y Matemáticas,

Universidad Austral de Chile,

Casilla 567, Valdivia, Chile.

Email: jorge.huentutripay@uach.cl 